\title{
OPEN Impact of monthly headache days on anxiety, depression and disability in migraine patients: results from the Spanish Atlas
}

\author{
P. Irimia ${ }^{1,2,3 凶}$, M. Garrido-Cumbrera ${ }^{4}$, S. Santos-Lasaosa ${ }^{3,5,6}$, M. Aguirre-Vazquez ${ }^{7}$, \\ J. Correa-Fernández ${ }^{4}$, I. Colomina ${ }^{9}$ \& P. Pozo-Rosich ${ }^{3,8,10}$
}

Identifying highly disabled patients or at high risk of psychiatric comorbidity is crucial for migraine management. The burden of migraine increases with headache frequency, but the number of headache days (HDs) per month after which disability becomes severe or the risk of anxiety and depression is higher has not been established. Here, we estimate the number of HDs per month after which migraine is associated with higher risk of anxiety and depression, severe disability and lower quality of life. We analysed 468 migraine patients (mean age $36.8 \pm 10.7 ; 90.2 \%$ female), of whom $38.5 \%$ had $\geq 15 \mathrm{HDs}$ per month. Our results show a positive linear correlation between the number of HDs per month and the risk of anxiety $(r=0.273 ; p<0.001)$, depression $(r=0.337 ; p<0.001)$ and severe disability $(r=0.519 ; p<0.001)$. The risk of anxiety is higher in patients having $\geq 3$ HDs per month, and those with $\geq 19 \mathrm{HD}$ ser month are at risk of depression. Moreover, patients suffering $\geq 10 \mathrm{HD}$ s per month have very severe disability. Our results suggest that migraine patients with $\geq 10 \mathrm{HDs}$ per month are very disabled and also that those with $\geq 3$ HDs per month should be screened for anxiety.

Migraine patients are usually classified into two fundamental categories: episodic (EM) and chronic migraine (CM). The established number of headache days (HDs) per month for differentiating episodic and CM is 15 days, according to the Third International Classification of Headache Disorders (ICHD-3) ${ }^{1}$. This division also implies differences between the two groups in disability, quality of life, the risk of comorbid diseases and the response to certain treatments ${ }^{2,3}$. However, the cut-off point of 15 monthly HDs was defined based on expert consensus, with recent studies showing that there is a substantial overlap in levels of burden, anxiety and depression between patients with frequent EM, and those with $\mathrm{CM}^{4-6}$. Thus, Torres-Ferrús, et al. ${ }^{4}$ reported that patients with 10-14 monthly HDs are as disabled as those with CM. In the same vein, Chalmer et al. ${ }^{5}$ studied a Danish-Russian cohort and observed that patients with migraine on 8-14 HDs per month are as disabled as those with ICHD-3 defined CM (15 or more HDs per month). They proposed that the cut-off point to distinguish EM and CM should be $\geq 8$ HDs per month. Furthermore, recent observations from the Chronic Migraine Epidemiology and Outcomes (CaMEO) study indicate a substantial overlap in the measures of burden and depression among respondents with 8-14 HDs per month and $\mathrm{CM}^{6}$. Moreover, Silberstein et al. ${ }^{7}$ have demonstrated that the number of HDs per month was correlated with a higher healthcare resource utilization and migraine burden.

Migraine is often associated with symptoms of depression and anxiety ${ }^{8-11}$, and headache frequency has been linked to a higher risk of anxiety and depression ${ }^{12-14}$. In addition, the presence of anxiety or depression increase the burden on individuals who experience migraine ${ }^{15,16}$. The clinician caring for patients with migraine should be able to identify those who are most disabled or at high risk of depression or anxiety. It can be hypothesized that there may be a number of HDs per month after which disability and quality of life clearly worsen or the risk of depression and anxiety is especially high. Nevertheless, the studies carried out to date do not allow us to establish

\footnotetext{
${ }^{1}$ Department of Neurology, Clínica Universidad de Navarra, Av. Pío XII 36, 31008 Pamplona, Spain. ${ }^{2}$ Navarra's Health Research Institute (IDISNA), Pamplona, Spain. ${ }^{3}$ Headache Study Group of the Spanish Neurological Society (GECSEN), Pamplona, Spain. ${ }^{4}$ Health and Territory Research (HTR), Universidad de Sevilla, Sevilla, Spain. ${ }^{5}$ Department of Neurology, Hospital Clínico Universitario Lozano Blesa, Zaragoza, Spain. ${ }^{6}$ Instituto de Investigación Sanitaria IIS Aragón, Zaragoza, Spain. ${ }^{7}$ Novartis Farmacéutica Spain, Barcelona, Spain. ${ }^{8}$ Headache Unit, Neurology Department, Vall d'Hebron University Hospital, Barcelona, Spain. ${ }^{9}$ Spanish Patient's Association of Migraine and Headache (AEMICE), Madrid, Spain. ${ }^{10}$ Headache Research Group, VHIR, Universitat Autónoma de Barcelona, Barcelona, Spain. ${ }^{凶}$ email: pirimia@unav.es
} 
the headache day threshold after which the burden of migraine is greater or there is a higher risk of comorbid psychiatric diseases. Thus, the objective of this analysis of the Spanish Migraine Atlas Survey is to define the number of HDs per month after which migraine is associated with a higher risk of anxiety and depression, severe disability and lower quality of life.

\section{Methods}

Study design. The Atlas of Migraine in Spain 2018 was an initiative of the Spanish Association of Migraine and Headache (AEMICE), carried out by the Health \& Territory Research (HTR) group of the University of Seville, in collaboration with the Study Group of Headaches of the Spanish Society of Neurology (GECSEN) and Novartis Spain.

Data source (Survey). A detailed questionnaire was prepared by the HTR research group, considering the opinion of a panel of neurologists, psychologists, research experts in headaches, and patients experiencing migraine. In order to establish and formulate the questions, a scientific literature review on headache was undertaken focusing on the following domains: diagnosis, comorbidity, healthcare utilization, pharmacological treatment, complementary therapies, employment and, social limitations. Additionally, we assessed the risk of anxiety and depression with the Hospital Anxiety and Depression Scale (HADS) ${ }^{17}, 18$, disability level with the Migraine Disability Assessment (MIDAS) ${ }^{19}$, and impact of migraine on quality of life with the Headache Needs Assessment (HANA ${ }^{20}$. A more detailed description of the methodology can be found in a previous study using the same sample ${ }^{21}$.

An online cross-sectional survey, with this questionnaire, was performed within the framework of the Spanish Migraine Atlas. The questionnaire was disseminated through the AEMICE patient association and patients filled it out voluntarily and anonymously. This survey was performed in full accordance with the Spanish law on data protection. As this was not an interventional study, no ethics committee approval was required according to the Spanish law 14/2007, 3 July, on biomedical research (BOE 159, 4 July 2007). However, all patients agreed to their participation through informed consent, and provided consent for aggregated reporting of research findings, before completing the survey. The survey was carried out between June and September 2017, including patients from all Spanish regions, using a non-probability sampling methodology. Of a total of 2,653 patients with migraine who began the questionnaire, after the validation, screening and cleaning process, the valid sample was made up of 1,283 patients. However, as one of the objectives of the present study was to assess the impact of the number of HDs per month on quality of life, psychiatric comorbidities and disability, we only included the patients who answered all questions including HADs, MIDAS, and HANA validated scales. We finally included 468 migraine patients. All patients included had been seen by a doctor within the last year and had received a medical diagnosis of migraine. The classification between CM and EM was established using the number of HDs reported by patients.

Indirect and direct cost were calculated from the data obtained in the survey. The direct costs related to medical visits, tests, and emergency room visits were obtained from the prices published in the Official Bulletins of the 17 Spanish Autonomous Communities. Average rates for 2017 were used due to the variability of prices between the different Autonomous Communities. Direct non-health care costs (borne by the patient) were self-reported by patients. Indirect cost was calculated from the patient's labour productivity losses due to medical visits, sick leave, and hospital admission days related to migraine in the economically active population. All costs were expressed in Euros referring to the year 2017, with the exception of the unit price per normal working hour in Spain, which was last updated in $2015^{22}$. The pharmacological costs were acquired from the economic study carried out in Spain in 2012 by Bloudek et al. ${ }^{23}$. The annual increase in the consumer price index of pharmaceutical products $^{24}$, was applied to the operating costs for 2010 used by Bloudek et al. ${ }^{23}$.

Variables. The questionnaire included variables related to healthcare utilization (diagnostic tests, medical visits, emergency visits, and hospital admissions), service utilization incurred by patients (visits to private specialists, and other complementary treatments for migraine) and data relating to patient work productivity losses in the previous year. In addition, three validated self-administered scales were used: HADS, MIDAS and HANA.

Patients were divided into four categories according to the frequency of HDs. Patients with 0-4 HDs per month were classified as low-frequency episodic migraine (LFEM), those with 5-8 HDs per month entered into the category of medium-frequency episodic migraine (MFEM), and when the number of HDs per month was between 9-14 HD per month the patients were classified as high-frequency episodic migraine (HFEM). Finally, patients with $\geq 15$ HD per month were included into the CM group.

HADS is an instrument designed for screening potential anxiety and depression rather than grading the severity of the anxiety and depression in the general population. The HADS questionnaire included 14 items, seven of which evaluate anxiety (HADS-A) and seven that evaluate depression (HADS-D). Each item is scored on a scale of 0-3, resulting in an overall score of 0-21 for both HADS-A and HADS-D to detect possible cases of depression and anxiety. According to the score obtained in the HADS scale it is possible to distinguish between no case: $0-7$; borderline case: $8-10$, and case: $11-21$ for both anxiety and depression ${ }^{17}$. The risk of anxiety and depression in HADS is considered from value 8 onwards ${ }^{18}$.

Additionally, disability was measured using the MIDAS scale, a 5-item questionnaire designed to evaluate disability within the past three months ${ }^{19}$. A score of 0-270 is used to indicate the overall level of disability due to headaches based on the following grading system: grade I, little or no disability (score of 0-5); grade II, mild disability (score of 6-10); grade III, moderate disability (score of 11-20); and grade IV, severe disability (score of $\geq 21$ ). The highest category is subdivided into grade IV-A, severe disability (scores of 21-40) and grade IV-B, very severe disability (scores of 41-270) ${ }^{19}$. 
HANA is a migraine-specific quality of life instrument measuring two dimensions of the chronic impact of migraine: frequency and bothersomeness. This scale contains the following seven domains: (i) anxiety/worry; (ii) depression/discouragement; (iii) self-control; (iv) energy; (v) function/work; (vi) family/social activities; and (vii) overall impact of migraine. The HANA validation studies confirmed internal consistency and reliability ${ }^{20}$.

Statistical analysis. A descriptive analysis of the population was completed through the mean and standard deviation for quantitative variables and the size and percentage for qualitative variables.

The Mann-Whitney test was used to compare the homogeneity of distribution between the number of HDs and variables with two categories. The Kruskal-Wallis test was used to compare the homogeneity of distribution between the number of HD and variable with more than two categories.

Partial correlation was used to evaluate the association between the frequency of HDs and the risk of anxiety, depression (HADS), disability (MIDAS), and quality of life (HANA) controlling by age, gender, education level, smoking, alcohol, BMI and health insurance coverage.

A scatter plot was used to represent the means of HADS anxiety, HADS depression, MIDAS and HANA versus the frequency of HD per month. To this graphical representation we added the linear trend lines for both the total and each of the established categories (LFEM, MFEM, HFEM and CM).

Predictions were made from simple linear regression equations at $95 \%$ confidence.

\section{Results}

Patient characteristics. The study included a sample of 468 migraine patients of whom 180 (38.5\%) had CM. The mean age of patients was $36.8( \pm 10.7)$ years and $90.2 \%$ were women. Clinical characteristics of patients with migraine are displayed in Table 1 . CT scans had been performed on $31.6 \%$ of the patients and MRIs on 26.3\%. Lumbar punctures had been carried out on $3.8 \%$ of the patients.

In addition, the mean number of visits to the emergency services due to migraine in the previous 12 months was $5.5( \pm 11.0)$, while the mean number of hospital admissions due to migraine in the same period was 4.8 $( \pm 19.5) .94 .6 \%$ had public health coverage (only or in combination with private).

The diagnostic delay in our sample was 6.6 years $(\mathrm{SD}=6.6$, Median $=5.0)$ and disease duration was 20.6 years $(\mathrm{SD}=11.6$, Median $=19.0) .81 .8 \%$ of patients took analgesics or anti-inflammatory drugs during a migraine attack and $23.7 \%$ took antidepressants as preventive treatment.

The annual direct health care cost per patient was $€ 3,054.25( \pm € 20,375.98)$, direct non-health care cost was $€ 1,600.55( \pm € 2,183.1)$, indirect costs were calculated at $€ 5,474.62( \pm € 7,273.65)$ and total cost was $€ 10,159.43$ $( \pm € 22,881.75)$ [Table 1].

According to the HADS scale, $69.0 \%$ were at risk of anxiety and $39.3 \%$ susceptible to depression. The average MIDAS score was $50.3( \pm 52.6)$ and the average HANA score was $96.5( \pm 34.5)$.

Headache frequency and the risk of anxiety, depression and severe disability. With respect to mental health, those at risk of anxiety reported a higher frequency of headache (13.2 vs 9.0; $\mathrm{p}<0.001$ ), as did those at risk of depression (14.8 vs 9.9; $\mathrm{p}<0.001)$. The HDs frequency was higher in patients with severe disability (MIDAS Grade I: 7.3 vs Grade II: 5.2 vs Grade III: 6.8 vs Grade IV-A: 10.3 vs Grade IV-B: 16.5; p $<0.001$ ) [Table 2].

Those patients with higher frequencies of HDs had a higher HADS anxiety mean score (LFEM: 7.9, MFEM: 9.8, HFEM: 9.9 and CM: 11.1; $\mathrm{p}<0.001$ ) and HADS depression (LFEM: 4.2, MFEM: 6.3, HFEM: 6.3 and CM: 8.4; $\mathrm{p}<0.001$ ). The MIDAS scale increased as the frequency of HDs' increased (LFEM: 17.7, MFEM: 34.3, HFEM: 40.3 and CM: 83.2; $\mathrm{p}<0.001$ ). The HANA scale increased as the frequency of HDs' increased (LFEM: 72.1, MFEM: 88.3, HFEM: 95.4 and CM: 116.1; $\mathrm{p}<0.001)$. Direct health care costs, direct non-health care costs, indirect costs and total costs were higher as the number of HD' increased $(p<0.001)$ [Table 3].

There was a positive linear correlation between the number of HDs and HADS anxiety $(\mathrm{r}=0.273 ; \mathrm{p}<0.001)$, HADS depression $(\mathrm{r}=0.337 ; \mathrm{p}<0.001)$, MIDAS scales $(\mathrm{r}=0.519 ; \mathrm{p}<0.001)$ and HANA $(\mathrm{r}=0.490 ; \mathrm{p}<0.001)$ and all are controlled by age, gender, education level, smoking, alcohol, BMI and health insurance coverage. Therefore, as the frequency of headaches increased, the risk of anxiety and depression increased, and the quality of life and disability of patients worsened [Table 4].

There was a positive linear trend between the number of HDs and risk of anxiety $\left(B=0.185 ; \mathrm{R}^{2}=0.380\right.$; $\mathrm{p}<0.001)$ [Fig. 1A], risk of depression $\left(B=0.198 ; \mathrm{R}^{2}=0.436 ; \mathrm{p}<0.001\right)$ [Fig. $1 \mathrm{~B}$ ], the HANA scale $(\mathrm{B}=1.863$; $\left.\mathrm{R}^{2}=0.617 ; \mathrm{p}<0.001\right)$ [Fig. 1C], and MIDAS scale $\left(\mathrm{B}=3.046 ; \mathrm{R}^{2}=0.565 ; \mathrm{p}<0.001\right)$ [Fig. 1D].

It was found that from the third day with a headache per month (MFEM), patients became part of the population at risk of anxiety, while the risk of depression appeared from day 19 (CM). From day 12 (HFEM), the values of the HANA were higher than the mean established at 96.5. Disability (MIDAS) was severe after suffering from three or more headache/days; and became very severe after 10 or more HDs/month.

\section{Discussion}

In the present study, the headache day threshold to identify migraine patients with severe disability is 10 HDs per month, and after 12 monthly HDs quality of life worsens above average. Our data are aligned with previous observations ${ }^{4,5,25}$ which have suggested that the headache day threshold after which migraine is associated with severe disability, lower quality of life disability, and high healthcare utilization in patients with migraine is below 15 HDs per month. Although our analysis was not predefined using a multiple regression analysis, we found a similar threshold reported by Torres-Ferrús et al. ${ }^{4}$ in a cohort of patients from a headache unit.

Anxiety and depression are common among migraine patients ${ }^{8-11}$ and impact on migraine-related disability and quality of life ${ }^{26}$. In our sample, we found that after three HDs per month anxiety impacts on migraine patients 


\begin{tabular}{|c|c|}
\hline Variables & Mean \pm SD or $\mathbf{n}(\%)$ \\
\hline \multicolumn{2}{|l|}{ Sociodemographic } \\
\hline Age & $36.8 \pm 10.7$ \\
\hline Gender. Female & $422(90.2)$ \\
\hline Marital status. Married or in relationship; N: 466 & $288(61.8)$ \\
\hline Educational level. University; $\mathrm{N}: 467$ & $250(53.5)$ \\
\hline \multicolumn{2}{|l|}{ Lifestyle habits } \\
\hline Smoking. Yes; N: 464 & $99(21.3)$ \\
\hline Alcohol. Yes & $73(15.6)$ \\
\hline BMI. Overweight or obesity; N: 467 & $166(35.6)$ \\
\hline \multicolumn{2}{|l|}{ Frequency of HDs } \\
\hline LFEM (0-4 days) & $108(23.1)$ \\
\hline MFEM (5-8 days) & $100(21.4)$ \\
\hline HFEM (9-14 days) & $80(17.1)$ \\
\hline CM (15-30 days) & $180(38.5)$ \\
\hline \multicolumn{2}{|l|}{ Healthcare utilization } \\
\hline CT-scan. Yes; N:405 & $129(31.9)$ \\
\hline Magnetic resonance imaging. Yes; N: 373 & $95(25.8)$ \\
\hline Lumbar puncture. Yes; N: 338 & $12(3.6)$ \\
\hline Number of emergency visits; N: 264 & $5.5 \pm 11.1$ \\
\hline Number of hospital admissions; N: 58 & $4.8 \pm 19.7$ \\
\hline Health Insurance. Public health; N: 467 & $442(94.6)$ \\
\hline \multicolumn{2}{|l|}{ Diagnostic delay } \\
\hline Years; N: 362 & $6.9 \pm 6.6$ \\
\hline \multicolumn{2}{|l|}{ Disease duration } \\
\hline Years; N: 466 & $20.6 \pm 11.6$ \\
\hline \multicolumn{2}{|l|}{ Treatment during a migraine attack } \\
\hline Analgesic or anti-inflammatory; N: 462 & $378(81.8)$ \\
\hline Triptan; N: 462 & $249(53.9)$ \\
\hline Other; $\mathrm{N}: 462$ & $112(24.2)$ \\
\hline \multicolumn{2}{|l|}{ Preventive treatments } \\
\hline Antidepressants; N: 443 & $105(23.7)$ \\
\hline Injections of botulinum toxin (type A Botox); N: 443 & $60(13.5)$ \\
\hline Anticonvulsant medications; N: 443 & $54(12.2)$ \\
\hline High blood pressure medication; N: 443 & $44(9.9)$ \\
\hline Minerals and vitamins; $\mathrm{N}: 443$ & $42(9.5)$ \\
\hline Other; N: 443 & $84(19.0)$ \\
\hline None preventive treatments; N: 443 & $196(44.2)$ \\
\hline \multicolumn{2}{|l|}{ Mental Health } \\
\hline HADS anxiety. Risk & $323(69.0)$ \\
\hline HADS depression. Risk & $184(39.3)$ \\
\hline \multicolumn{2}{|l|}{ Disability } \\
\hline MIDAS & $50.3 \pm 52.6$ \\
\hline \multicolumn{2}{|l|}{ Quality of life } \\
\hline HANA & $96.5 \pm 34.5$ \\
\hline \multicolumn{2}{|l|}{ Societal costs (in Euros) } \\
\hline Direct health care cost & $3,054.25 \pm 20,375.98$ \\
\hline Direct non healthcare cost & $1,600.55 \pm 2,183.1$ \\
\hline Indirect cost & $5,474.62 \pm 7,273.65$ \\
\hline Total cost & $10,159.43 \pm 22,881.75$ \\
\hline
\end{tabular}

Table 1. Sociodemographic characteristics, Patient-reported Outcomes (HADS, HANA and MIDAS) \& Healthcare Resource Utilization ( $\mathrm{N}=468$, unless otherwise specified). PRO patient-reported outcome; $H A D S$ hospital anxiety and depression scale; HANA headache needs assessment; MIDAS migraine disability assessment scale; LFEM (Low-frequency episodic migraine); MFEM (Medium-frequency episodic migraine); HFEM (High-frequency episodic migraine); CM (Chronic migraine). 


\begin{tabular}{|c|c|c|c|}
\hline \multicolumn{2}{|l|}{ Variables } & $\begin{array}{l}\text { Number of HD per month } \\
\text { Mean } \pm \text { SD } / \mathrm{r} \text { correlation }\end{array}$ & P-value \\
\hline \multicolumn{4}{|l|}{ Sociodemographic } \\
\hline \multirow{4}{*}{ Age categories } & $16-31$ & $12.2 \pm 8.3$ & \multirow{4}{*}{0.383} \\
\hline & $32-47$ & $10.8 \pm 7.2$ & \\
\hline & $48-64$ & $12.4 \pm 8.2$ & \\
\hline & $\geq 65$ & $13.0 \pm 9.8$ & \\
\hline \multirow{2}{*}{ Gender } & Male & $11.6 \pm 9.4$ & \multirow{2}{*}{0.490} \\
\hline & Female & $11.6 \pm 7.6$ & \\
\hline \multirow{4}{*}{ Marital status } & Single & $11.3 \pm 8.1$ & \multirow{4}{*}{0.173} \\
\hline & Married/relationship & $11.4 \pm 7.5$ & \\
\hline & Divorced/separated & $15.7 \pm 9.4$ & \\
\hline & Widow & $6.0 \pm 0.0$ & \\
\hline \multirow{4}{*}{ Educational level } & No schooling & $17.0 \pm 12.4$ & \multirow{4}{*}{$0.020^{*}$} \\
\hline & Primary school & $15.1 \pm 10.0$ & \\
\hline & Secondary school & $12.4 \pm 8.0$ & \\
\hline & University & $10.5 \pm 7.2$ & \\
\hline \multicolumn{4}{|l|}{ Lifestyle habits } \\
\hline \multirow{2}{*}{ Smoking } & Yes & $13.4 \pm 8.2$ & \multirow{2}{*}{$0.007^{\star}$} \\
\hline & No & $11.1 \pm 7.7$ & \\
\hline \multirow{2}{*}{ Alcohol } & Yes & $9.1 \pm 7.2$ & \multirow{2}{*}{$0.002^{\star}$} \\
\hline & No & $12.1 \pm 7.9$ & \\
\hline \multirow{4}{*}{ BMI } & Underweight & $11.5 \pm 7.7$ & \multirow{4}{*}{0.541} \\
\hline & Normal weight & $11.7 \pm 7.6$ & \\
\hline & Overweight & $11.9 \pm 8.3$ & \\
\hline & Obesity & $10.5 \pm 8.1$ & \\
\hline \multicolumn{4}{|l|}{ Healthcare use } \\
\hline \multirow{2}{*}{ Scanner or CT-scan } & Yes & $14.3 \pm 8.4$ & \multirow{2}{*}{$<0.001^{\star}$} \\
\hline & No & $10.7 \pm 7.3$ & \\
\hline \multirow{2}{*}{ Magnetic resonance imaging } & Yes & $14.7 \pm 8.5$ & \multirow{2}{*}{$<0.001^{\star}$} \\
\hline & No & $10.6 \pm 7.2$ & \\
\hline \multirow{2}{*}{ Lumbar or spinal puncture } & Yes & $14.8 \pm 8.5$ & 0118 \\
\hline & No & $11.1 \pm 7.6$ & 0.118 \\
\hline & Public & $11.6 \pm 8.0$ & \\
\hline Health Insurance Coverage & Private & $13.6 \pm 8.8$ & 0.486 \\
\hline & Public and private combination & $11.0 \pm 7.2$ & \\
\hline Psychological health & & & \\
\hline HADS Anxiety & No risk & $8.9 \pm 6.5$ & $<0.001^{*}$ \\
\hline & Risk & $12.8 \pm 8.1$ & \\
\hline HADS Denression & No risk & $9.9 \pm 7.0$ & $<0001 *$ \\
\hline HADS Depiession & Risk & $14.2 \pm 8.4$ & -0.001 \\
\hline Disability & & & \\
\hline & Grade I & $7.3 \pm 7.6$ & \\
\hline & Grade II & $5.2 \pm 3.6$ & \\
\hline MIDAS & Grade III & $6.8 \pm 5.9$ & $<0.001^{\star}$ \\
\hline & Grade IV-A & $10.3 \pm 6.5$ & \\
\hline & Grade IV-B & $16.5 \pm 7.1$ & \\
\hline Quality of life & & & \\
\hline HANA & HANA total & 0.520 & $<0_{0.001}^{*}$ \\
\hline
\end{tabular}

Table 2. Bivariate analysis between the number of HD per month and sociodemographic, life habits, healthcare utilization, mental health and disability. PRO patient-reported outcome; HADS hospital anxiety and depression scale; HANA headache needs assessment; MIDAS migraine disability assessment scale; LFEM (Low-frequency episodic migraine); MFEM (Medium-frequency episodic migraine); HFEM (High-frequency episodic migraine); $C M$ (Chronic migraine). 


\begin{tabular}{|c|c|c|c|c|c|}
\hline & \multicolumn{4}{|l|}{ Mean \pm SD } & \multirow[b]{3}{*}{ P-value } \\
\hline & \multicolumn{4}{|l|}{ Number of HD } & \\
\hline & LFEM N: 108 & MFEM N: 100 & HFEM N: 80 & CM N: 180 & \\
\hline HADS Anxiety & $7.9 \pm 3.4$ & $9.8 \pm 4.2$ & $9.9 \pm 3.9$ & $11.1 \pm 4.1$ & $<0.001^{*}$ \\
\hline HADS Depression & $4.2 \pm 3.5$ & $6.3 \pm 4.0$ & $6.3 \pm 4.1$ & $8.4 \pm 4.7$ & $<0.001^{*}$ \\
\hline MIDAS & $17.7 \pm 15.6$ & $34.3 \pm 31.9$ & $40.3 \pm 28.4$ & $83.2 \pm 65.1$ & $<0.001^{\star}$ \\
\hline HANA & $72.1 \pm 31.9$ & $88.3 \pm 29.3$ & $95.4 \pm 31.1$ & $116.1 \pm 28.6$ & $<0.001^{\star}$ \\
\hline DHC & $888.7 \pm 1,645.2$ & $1,582.1 \pm 2,676.7$ & $1,090.7 \pm 1,159.6$ & $6,044.9 \pm 32,592.1$ & $<0.001^{*}$ \\
\hline DNHC & $888.9 \pm 1,037.1$ & $1,158.0 \pm 1,169.1$ & $1,496.3 \pm 1,909.1$ & $2,397.8 \pm 2,894.5$ & $<0.001^{*}$ \\
\hline IC & $2,106.1 \pm 3,839.8$ & $3,794.3 \pm 4,957.0$ & $6,423.9 \pm 6,754.7$ & $8,007.4 \pm 8,956.1$ & $<0.001^{*}$ \\
\hline TC & $3,882.4 \pm 4,812.6$ & $6,534.4 \pm 6,046.4$ & $9,010.9 \pm 7,846.2$ & $16,450.1 \pm 35,117.5$ & $<0.001^{*}$ \\
\hline
\end{tabular}

Table 3. Bivariate analysis between the number of $\mathrm{HD}$ and mental health, disability and cost. LFEM lowfrequency episodic migraine; MFEM medium-frequency episodic migraine; HFEM high-frequency episodic migraine; $C M$ chronic migraine; $D H C$ direct health care cost; $D N H C$ direct non-health care cost; IC indirect cost; TC total cost.

\begin{tabular}{|l|l|c|}
\hline & r correlation & p-value \\
\hline HADS Anxiety & 0.273 & $<\mathbf{0 . 0 0 1}^{*}$ \\
\hline HADS Depression & 0.337 & $<\mathbf{0 . 0 0 1}^{*}$ \\
\hline MIDAS & 0.519 & $<\mathbf{0 . 0 0 1}^{*}$ \\
\hline HANA & 0.490 & $<\mathbf{0 . 0 0 1}^{*}$ \\
\hline
\end{tabular}

Table 4. Partial correlation between the number of HD and healthcare utilization, mental health and disability. ${ }^{\star}$ Controlling by age, gender, education level, smoking, alcohol, BMI and health insurance coverage.

and interfere in everyday life. Headache frequency was previously associated with higher scores on the HADS ${ }^{27}$. However, anxiety may impact patients with LFEM, as observed here. Contrary to our observation, Zebenholzer et al. ${ }^{15}$ did not find an increased risk of anxiety in migraine patients with LFEM. Interictal anxiety is an important component of the burden of EM especially in those patients with severe migraine attacks ${ }^{28}$. The uncertainty of not knowing when the next migraine will occur and the severity of the attack may cause anxiety symptoms such as restlessness, persistent worrying and inability to concentrate, among others ${ }^{29}$. Moreover, migraine patients often endorse higher levels of neuroticism and are more likely than average to experience anxiety ${ }^{30,31}$. Early recognition and management of anxiety may be of great value to improve the life of migraine patients, and, according to our findings, screening of anxiety should be considered as part of the routine clinical evaluation in patients with migraine, including those with LFEM. In this respect, the use of preventive medication should be considered when the frequency of attacks per month is two or higher, but particularly in those patients with severe disability or comorbidities such as anxiety ${ }^{6,32}$.

Additionally, we studied the number of HDs after which patients have a higher risk of depression. Surprisingly, the headache day threshold from which a migraine patient has a high risk for depression is 19 monthly HDs. Different studies have shown that the risk of depression is increased in patients with frequent migraine attacks $^{13,15,33}$. Furthermore, depression is a risk factor for migraine chronification ${ }^{34}$. In contrast with our results, previous observations suggest that migraine patients are at risk of depression when the cut-off point is somewhat below 15 HDs per month ${ }^{5,6,25}$. Multiple reasons explain why the headache threshold is so different for anxiety and depression in the present study. First, depression onset is not associated with minor episodic stressors, but to chronic stress or major life events ${ }^{35}$. Nineteen days per month means that patients are spending more than half their life with pain and disability, and gives weight to the consideration of depression as secondary. That realization surely will lead to hopelessness and the inability to put in place appropriate pain-coping strategies that characterize depression in migraine patients ${ }^{36}$. Also, the relationship between migraine and depression is complex and bidirectional ${ }^{9}$. Migraine may aggravate depressive symptoms and is associated with a lower improvement in $\operatorname{mood}^{37}$. Finally, we cannot exclude that the high headache day threshold for depression observed here is because the HADS scale only captures a significant change in those patients in whom depressive symptoms are very severe.

Among the strengths of the present study was the use of a large sample including 468 migraine patients who were evaluated using validated scales to measure anxiety, depression, quality of life and disability. However, this study is subject to several limitations. First, the diagnosis of anxiety and depression was based on the patient's response and self-reported validated questionnaires and not in-person interviews, so the clinical diagnosis cannot be verified. In addition, although HADS is widely used for detecting anxiety and depressive disorders ${ }^{12}$, this scale is a screening tool rather than a diagnostic test. Another limitation is that the survey was completed through an online platform, and respondents had to have access to internet, as well as knowledge of technology. In addition, 


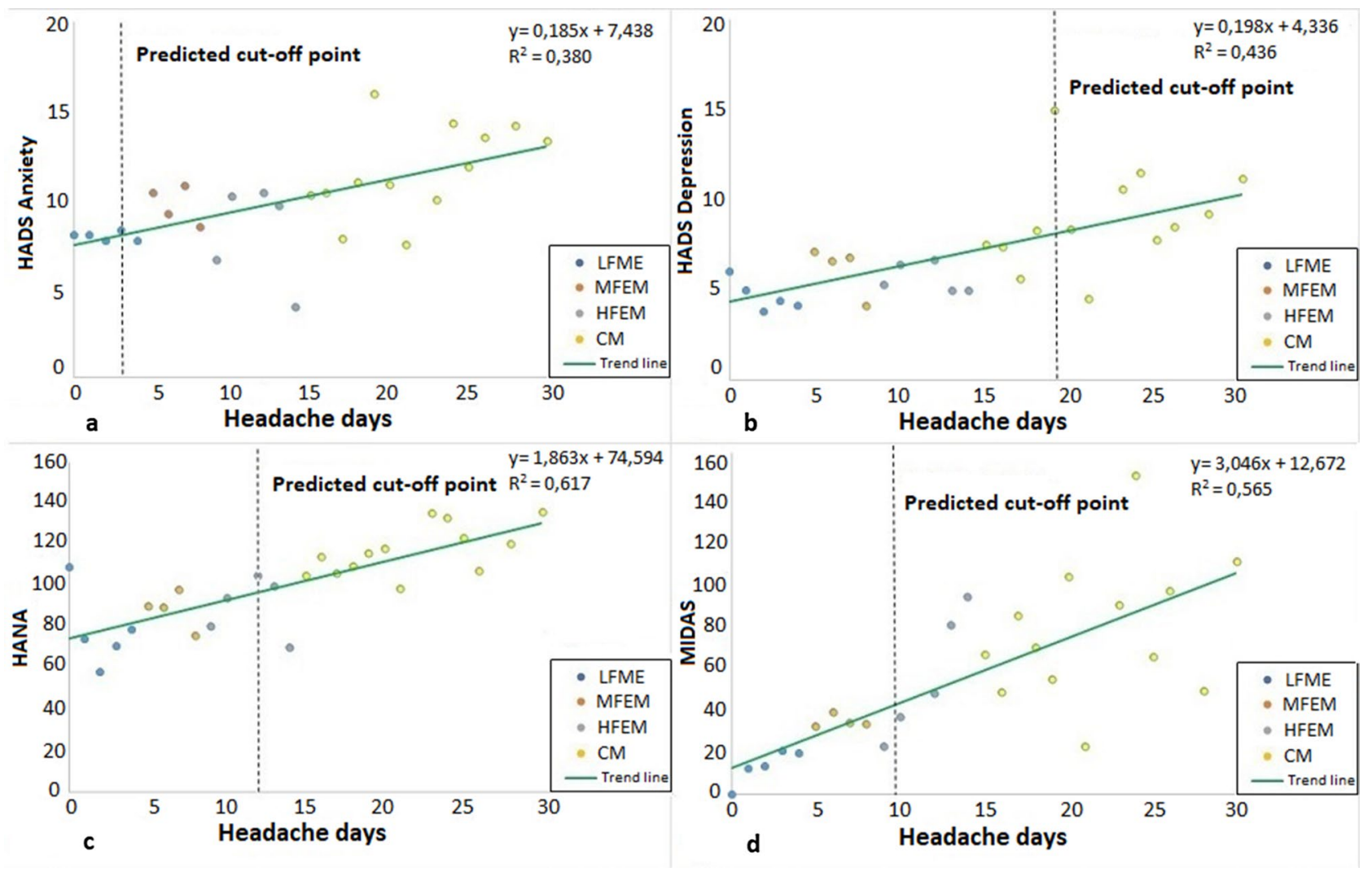

Figure 1. Scatter plot between anxiety, depression, quality of life, disability and number of days with headache. HADS: Hospital Anxiety and Depression Scale; HANA: Headache Needs Assessment; MIDAS: Migraine Disability Assessment Scale; LFEM (Low-frequency episodic migraine); MFEM (Medium-frequency episodic migraine); HFEM (High-frequency episodic migraine); CM (Chronic migraine).

a high proportion of patients were invited to participate in the survey through the association AEMICE, so patients with severe forms of migraine may be over-represented.

The increase in the number of HDs per month is associated with high disability, and a decrease in the quality of life. Anxiety may have a significant impact on migraine patients when the number of HDs is relatively low whilst depression strikes later within the headache day threshold. Our results support the need to redefine the diagnostic criteria of CM to include those patients with less than 15 monthly HDs but with high disease burden and treatment needs.

Data availability

The data that support the findings of this study are available from the corresponding author upon reasonable request.

Received: 19 December 2020; Accepted: 23 March 2021

Published online: 15 April 2021

\section{References}

1. Olsen, J. The International Classification of Headache Disorders, 3rd edition. Cephalalgia 38(1), 1-211 (2018).

2. Katsarava Z, Buse DC, Manack AN, Lipton RB. Defining the differences between episodic migraine and chronic migraine. Vol. 16, Current Pain and Headache Reports. Curr Pain Headache Rep; 2012. p. 86-92.

3. Aurora SK. Is chronic migraine one end of a spectrum of migraine or a separate entity? Vol. 29, Cephalalgia. Cephalalgia; 2009. p. 597-605.

4. Torres-Ferrús, M., Quintana, M., Fernandez-Morales, J., Alvarez-Sabin, J. \& Pozo-Rosich, P. When does chronic migraine strike? A clinical comparison of migraine according to the headache days suffered per month. Cephalalgia 37(2), 104-113 (2017).

5. Chalmer, M. A. et al. Proposed new diagnostic criteria for chronic migraine. Cephalalgia 40(4), 399-406 (2020).

6. Lipton RB, Reed ML, Fanning KM, Buse DC, Goadsby PJ, Olesen J, et al. Exploring the Boundaries Between Episodic and Chronic Migraine: Results from the Cameo Study. Vol. 60, 62nd Annual Scientific Meeting American Headache Society. 2020. p. 59.

7. Silberstein, S. D. et al. Health care resource utilization and migraine disability along the migraine continuum among patients treated for migraine. Headache J. Head Face Pain. 58(10), 1579-1592 (2018).

8. Radat, F. \& Swendsen, J. Psychiatric comorbidity in migraine: A review. Cephalalgia 25(3), 165-178 (2005).

9. Antonaci, F. et al. Migraine and psychiatric comorbidity: a review of clinical findings. J. Headache Pain. 12(2), 115-125 (2011).

10. Dresler, T. et al. Understanding the nature of psychiatric comorbidity in migraine: A systematic review focused on interactions and treatment implications. J. Headache Pain. 20(1), 1-17 (2019).

11. Lampl, C. et al. Headache, depression and anxiety: associations in the Eurolight project. J. Headache Pain. 17(1), 1 (2016). 
12. Karimi, L. et al. The prevalence of migraine with anxiety among genders. Front Neurol. 26(11), 569405 (2020).

13. Blumenfeld, A. M. et al. Disability, HRQoL and resource use among chronic and episodic migraineurs: results from the International Burden of Migraine Study (IBMS). Cephalalgia 31(3), 301-315 (2011).

14. Zwart, J. A. et al. Depression and anxiety disorders associated with headache frequency: The Nord-Trøndelag Health Study. Eur. J. Neurol. 10(2), 147-152 (2003).

15. Zebenholzer, K. et al. Impact of depression and anxiety on burden and management of episodic and chronic headaches-a crosssectional multicentre study in eight Austrian headache centres. J. Headache Pain. 17(1), 1-10 (2016).

16. Pesa, J. \& Lage, M. J. The Medical Costs of Migraine and Comorbid Anxiety and Depression. Headache J. Head Face Pain. 44(6), $562-570$ (2004).

17. Zigmond, A. S. \& Snaith, R. P. The Hospital Anxiety and Depression Scale. Acta Psychiatr. Scand. 67, 361-370 (1983).

18. Barczak, P. et al. Patterns of psychiatric morbidity in a genito-urinary clinic: A validation of the hospital anxiety depression scale (HAD). Br. J. Psychiatry. 152, 698-700 (1988).

19. Stewart, W. F. et al. An international study to assess reliability of the Migraine Disability Assessment (MIDAS) score. Neurology 53(5), 988-994 (1999).

20. Cramer, J. A., Silberstein, S. D. \& Winner, P. Development and validation of the headache needs assessment (HANA) survey. Headache J Head Face Pain. 41(4), 402-409 (2001).

21. Irimia, P. et al. Estimating the Savings of a Migraine Free Life: Results from the Spanish Atlas. Eur J Neurol. 1, 1 (2020).

22. Instituto Nacional de Estadística (INE). Número medio de horas efectivas semanales trabajadas por todos los ocupados (hayan o no trabajado en la semana) por situación profesional, sexo y ocupación (empleo principal). Encuesta de Población Activa $2015 T 3$. 2015.

23. Bloudek, L. M. et al. Cost of healthcare for patients with migraine in five European countries: results from the International Burden of Migraine Study (IBMS). J Headache Pain. 13(5), 361-378 (2012).

24. Instituto Nacional de Estadística(INE). Índice de Precios de Consumo (Productos farmacéuticos) de 2009 a 2017. Base 2016. 2017

25. Ruscheweyh, R., Müller, M., Blum, B. \& Straube, A. Correlation of headache frequency and psychosocial impairment in migraine: A cross-sectional study. Headache 54(5), 861-871 (2014).

26. Lantéri-Minet, M., Radat, F., Chautard, M. H. \& Lucas, C. Anxiety and depression associated with migraine: influence on migraine subjects' disability and quality of life, and acute migraine management. Pain 118(3), 319-326 (2005).

27. Te, ChuH. et al. Associations between depression/anxiety and headache frequency in Migraineurs: a cross-sectional study. Headache 58(3), 407-415 (2018)

28. Lampl, C. et al. Interictal burden attributable to episodic headache: findings from the Eurolight project. J Headache Pain. 17(1), $1-10$ (2016).

29. Peres, M. F. P., Mercante, J. P. P., Tobo, P. R., Kamei, H. \& Bigal, M. E. Anxiety and depression symptoms and migraine: a symptombased approach research. J Headache Pain. 18(1), 1 (2017).

30. Davis, R. E., Smitherman, T. A. \& Baskin, S. M. Personality traits, personality disorders, and migraine: a review. Neurol Sci 34, 1 (2013).

31. Silberstein, S., Lipton, R. \& Breslau, N. Migraine: association with personality characteristics and psychopathology. Cephalalgia 15(5), 358-369 (1995).

32. Evers, S. et al. EFNS guideline on the drug treatment of migraine-Revised report of an EFNS task force. Eur. J. Neurol. 16, 968-981 (2009).

33. Buse, D. C., Manack, A., Serrano, D., Turkel, C. \& Lipton, R. B. Sociodemographic and comorbidity profiles of chronic migraine and episodic migraine sufferers. J. Neurol. Neurosurg. Psychiatry. 81(4), 428-432 (2010).

34. Ashina, S. et al. Depression and risk of transformation of episodic to chronic migraine. J. Headache PainHeadache Pain. 13, 615-624 (2012).

35. Vrshek-Schallhorn, S. et al. Chronic and episodic interpersonal stress as statistically unique predictors of depression in two samples of emerging adults. J. Abnorm. Psychol. 124(4), 918-932 (2015).

36. Pompili M, Serafini G, di Cosimo D, Dominici G, Innamorati M, Lester D, et al. Psychiatric comorbidity and suicide risk in patients with chronic migraine. Vol. 6, Neuropsychiatric Disease and Treatment. Dove Medical Press Ltd.; 2010. p. 81-91.

37. Hung C-I, Liu C-Y, Yang C-H, Wang S-J. The Impacts of Migraine among Outpatients with Major Depressive Disorder at a TwoYear Follow-Up. Stewart R, editor. PLoS One. 2015 May 22;10(5):e0128087.

\section{Acknowledgements}

We acknowledge the patients for their participation in this study and to the Spanish patient's headache association (AEMICE) for its collaboration. This study has been supported by Novartis Farmacéutica Spain.

\section{Author contributions}

Conceptualization, P.I., S.S.-L. and P.P.-R.; methodology, P.I., M.G.-C., S.S.-L., P.P.-R., and J.C.-F.; formal analysis, J.C.-F.; investigation, P.I., P.P.-R., M.G.-C., S.S.-L. and J.C.-F.; writing-original draft preparation, M.G.-C. and J.C.-F.; writing-review and editing, P.I., P.P.-R., M.G.-C., S.S.-L., J.C.-F., I.C. and M.A.-V.; supervision, M.G.-C., P.I., P.P.-R., S.S.-L., J.C-F. and M.A.-V. All authors have read and agreed to the published version of the manuscript.

\section{Competing interests}

The authors declare no competing interests.

\section{Additional information}

Correspondence and requests for materials should be addressed to P.I.

Reprints and permissions information is available at www.nature.com/reprints.

Publisher's note Springer Nature remains neutral with regard to jurisdictional claims in published maps and institutional affiliations. 
(c) (i) Open Access This article is licensed under a Creative Commons Attribution 4.0 International cc) License, which permits use, sharing, adaptation, distribution and reproduction in any medium or format, as long as you give appropriate credit to the original author(s) and the source, provide a link to the Creative Commons licence, and indicate if changes were made. The images or other third party material in this article are included in the article's Creative Commons licence, unless indicated otherwise in a credit line to the material. If material is not included in the article's Creative Commons licence and your intended use is not permitted by statutory regulation or exceeds the permitted use, you will need to obtain permission directly from the copyright holder. To view a copy of this licence, visit http://creativecommons.org/licenses/by/4.0/.

(C) The Author(s) 2021 